\title{
Semantic Method of Recovering of Medical Articles
}

\author{
Jucélio Costa de Araújo \\ Federal Institute of Goiás \\ Avenida Furnas n.55 \\ Itumbiara, Brasil
}

\author{
José Maria Parente de \\ Oliveira \\ Aeronautics Institute of Technology \\ Praça Marechal Eduardo Gomes n. \\ 50 São José dos Campos, Brasil
}

\author{
Leonardo Garcia Marques \\ Federal Institute of Goiás \\ Avenida Furnas n.55 \\ Itumbiara, Brasil
}

\begin{abstract}
The amount of information produced today reached an unprecedented contingent in history, which makes it difficult to locate relevant documents in a search. This situation leads to the need to use tools that facilitate the search, making information retrieval area increasingly important in the development of this context. Based on the organization of documents and ontology, information retrieval area has the challenge of getting smarter ways to recovery than just select syntactically documents, exploiting to the full the semantic context when selecting the information. This paper presents a semantic enrichment method that seeks to improve the quality of results when querying a database of medical articles. The proposed method performs a search on the repository of articles that is submitted to latent semantic analysis together with the National Cancer Institute (NCI) ontology and the lexical WordNet ontology database. After this joint treatment, the semantic relationship of those new terms to the survey conducted in the context is performed in order to improve the accuracy of recovery and enabling the retrieve of more relevant articles regarding the search.
\end{abstract}

\section{Keywords}

Information Retrieval, Semantic Enrichment, Ontology, Comparison by Similarity.

\section{INTRODUCTION}

Currently, the amount of information that is produced is growing every day, reaching large proportions. This large number of available data, is hindering the process of location information, especially those considered relevant in conducted searches, making their recovery is not optimal. Below I describe some of the notorious problems that occur during the searches done on the Web: Undertake all types of searches, before obtaining a satisfactory result, occurring with this, a large consumption of time, Search engines can not identify the semantics of a desired search, bringing results, often not relevant, You get tired enough in handling searches, and often give up in the middle of a search, the complexity in which it becomes, failing to reach the desired goal.

This scenario then ratifies the need to have efficient mechanisms to search for recovery and organizing data so that they can maximize their reuse in a simple and efficient way. So the Information Retrieval (IR) is now an activity of great importance to the extent that it became one of the most important actors in the rapid development of new information and communication technologies. It must be possible, among the large volume of documents available, finding those that best fit our needs in the shortest time. Consequently several questions arise about these information retrieval tools, particularly in terms of their performance and the relevance of the results that they offer. Thus the use of semantics in searches is growing every day, and becomes the subject of studies in the search for better results in the recovery information semantics is necessary in order to best perform certain tasks in certain areas, however, depending on the task, it is not necessary that the semantics be extremely detailed regarding the formation of concepts and semantic relations, since the semantics is an auxiliary item to the task. Traditional methods of search in medical ontologies can not always get results free of irrelevant content because the large amount of information retrieved does not always reach the intended objectives to the survey, making it difficult to separate the recovery information that was retrieved in a timely manner. Thus, there has been the need to create tools that are capable of producing significant results, taking into account not only the query syntax, but also its semantics, allowing us to better contextualized searches. Then comes the need to develop new methods using the various technologies.

There are several research approaches in this area. Works as [1] in the medical field, use thesaurus in semantic recovery strategies. Broader approaches, not specific to a particular area of knowledge, often require the implementation of semantic similarity analysis techniques [2] the combination of the use of thesauri to ontology [3]. [4] show, for example, how ontologies can be used as a basis for semantic retrieval of objects based on specific learner's characteristics, leading to adaptive strategies for teaching and learning.

The proposed Article introduces a semantic enrichment model based on a similarity matrix , and use of Latent Semantic to make the system learn to recognize the context in which it is being inserted and, from there, establish relevant semantic relationships between terms searched in repositories of medical supplies, leading to more precise and contextualized results.

\section{SEMANTIC WEB}

The Web is already an integral part of everyday life for most people. Its first generation was based on static pages, individually made by hand, and the subsequent generation was made up of dynamic pages, with the major contributors the users, who make them more participatory. These generations have benefited from the simplicity of HTML (Hypertext Markup Language - Hypertext Markup Language), but also had to face their limitations and seek innovations to solve issues for which the language did not support.

The Semantic Web describes a qualitatively different from the current one, in which the contents, instead of being structured to be displayed and read by persons should have a "intelligible" semantics for programs, called "software agents"; such programs that are not specifically designed to process only a certain type of data. 
According to [5], the Semantic Web is not a separate Web but an extension of the current Web in which information brings some well-defined meaning, allowing people and computers having better conditions to work in cooperation . In this context, the semantic description of a vocabulary is crucial as it provides a broad understanding of the characteristics and properties of its classes and relationships between them. This can be achieved by ontologies.

\section{ONTOLOGY AND WORDNET}

In computer science, ontology is a formal conceptualization of a domain. According [6] usually it specifies the classes of objects that exist, relations between classes, the possible relations between instances of classes and restrictions on such cases. An ontology defines terms that denoting these classes and relationships as well as individual objects and defines the rules governing the combination of terms and relationships in a knowledge domain [7]. They are, therefore, conceptual models that capture and explain the vocabulary used in applications semantics [8].

Ontologies can be represented by a OWL scheme, made up of classes with properties, definition of instances, declaration of properties and interpretation of the logical relationship between classes and instances permitted by the formal semantics. Various tools now offer support for the use of OWL, as this language provides resources for the creation and manipulation of an ontology, and it is recommended by the $\mathrm{W} 3 \mathrm{C}$.

The WordNet is a lexical database, which has the synonym the primary relationship between words, was first proposed [9] in the 80s and consists of a large bank of English Lexicon data, grouping nouns, verbs, adjectives and adverbs into sets of cognitive synonyms (synsets) that express different concepts, contains information about words, compound words, verbs, idiomatic phrases, hierarchical relationships between words, allowing the use of system for information retrieval, categorization of texts, inference, coherent texts and connecting to the Semantic Web among other properties. Several academic applications have been created using the WordNet, including quoted information retrieval based on knowledge bases [10], image recovery through descriptions [11] and categorization of texts [12]. In the process of formation of ontologies, a tool that stands out is the WordNet, which is freely and publicly available for download, very useful for computational linguistics and natural language processing [13]. It makes possible not only the retrieval of information, but also the analysis of the meaning of sentences.

\section{INFORMATION RETRIEVING}

The Information Retrieval (IR) is the science devoted to finding relevant documents, not just the mere matching and standards identification[14]. It is a science to find documents, extracting information inside of documents, create metadata about them, both in SGDBs, and in collections such as on the WEB. Information retrieval is a dedicated area to facilitate document retrieval and presentation orderly according to relevance criteria. As the volume of information grows and the models currently used in the recovery prove ineffective, it is evident the need to propose and test more complex document templates and forms more elaborate queries.

In 1983, [15] one of the pioneers in the field of Information Retrieval, predicted that come the time when people would be forced to use information more easily available and ignore the rest of it. And this is actually happening because old limited and expensive corporative systems have been replaced by free search systems, for example, the giants Google and Yahoo. Inside them, the trivial information on a given subject can be found, but it is difficult to determine whether the result of the query made is sufficient or relevant because as it grows the amount of web pages, it becomes increasingly difficult to find quality information and Information Retrieval models in use are shown inadequate and need improvement.

Based on the organization of documents and ontologies, information retrieval area has the challenge of more intelligent forms of recovery than just select syntactically documents, exploiting to the full the semantic context when selecting the information.

Medicine and health are areas suitable for the use of data recovery [16]. The information retrieved should be better handled in this specific area, as they will influence the strategies to be defined by doctors. Therefore, this paper aims to treat the specific problem of data recovery in the medical field, by creating a model based on recovery of medical devices using semantic enrichment.

\section{RELATED WORK}

The work presented here supports the use of a semantic enrichment method for medical articles queries, along with detection of negative expressions and semantic annotation texts in order to improve the accuracy indices in the search procedures in medical documents.Some jobs are related in relation to medical searches, as work below.

[17] presented an approach to retrieve relevant articles on a biomedical corpus. The authors They considered four types of operators to expand search user from the MeSH ontology. The operators were used for setting the weights of a query, prioritize and rank the results research. Phrases found in the base containing all terms. They have higher weights than those that do not contain. The synonym list found in the ontology was used to expand the search user.

[18] has developed rules for extraction medical information in databases. This work was created a special ontology which represents the concepts in two models: one to represent the hierarchical structures and other dedicated grammars to process documents and fill in the template provided by proposed model. The authors developed techniques for linguistic extract information from mammary tissues and pathological diagnoses in Mammograms reports. In this work rules are designed to extraction of grammatical terms as ambiguous words , expressions negative, tokenization texts and anaphora expressions.

[19] identifies relevant terms electronic documents from the recognition process named entities. The goal is to write down the class instances biological from abstracts or full texts within the library PubMed. This model also features a semantic index documents and terms found. The technique is used to extract information from medical libraries, preprocess documents and applying a lexicon to perform the recognition named entities. The study presented by the authors allowed significantly reduce the number of irrelevant documents without loss of relevant documents

\section{ENRICHMENT METHOD}

The semantic enrichment of English Semantic Augmentation, is the process of attaching semantic concepts to specific parts of a text, providing a framework for 
automatic interpretation of its meaning and understanding of the domain [20]. For this, the identification is performed and the mapping of key terms from the textual content by PLN [21]. These terms are semantically associated with concepts from a domain ontology, allowing the understanding of the content [22]. These semantic relationships allow complex exploration and the discovery of information about a particular resource [23].

Enrich a query is to add words that increase your power of expression. Lexical databases (such as WordNet) could in the beginning, being employed for this purpose. However, not always obtains terms that comply with the query context, and this is the great advantage of the proposed model.

\subsection{Proposed Model}

The semantic enrichment method proposed in this paper is to make the system to recognize the context in which it appears

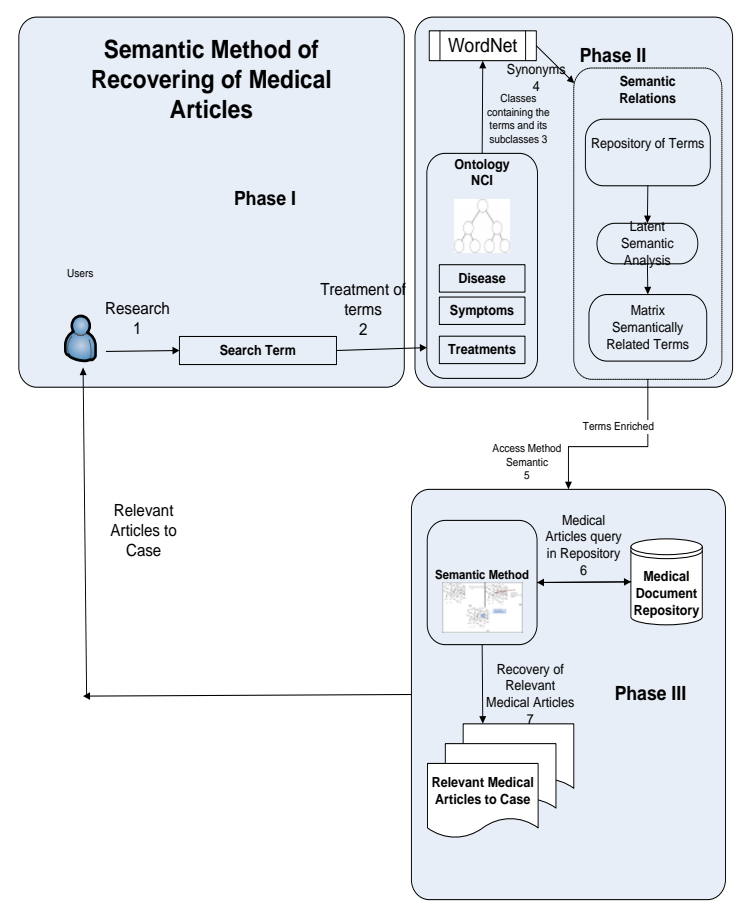

Fig. 1. Phases for the semantic enrichment of term

All terms that make up the names of the selected classes form a first set of terms - element (2) of Fig. 1. At this point, it looks up for classes (and associated subclasses) whose names feature in their formation the term to be enriched. The termt elements are submitted to Matrix Semantic Similarity (MSS), which contains a collection of terms belonging to the area of knowledge in which it is working. The MSS is a square matrix, with each line and each column representing a term. It has property to indicate the percentage of semantic similarity between a term of collection and any other term. Thus, the MSSij element is a numerical value between 0 and 1 which represents the degree of semantic similarity between $i$ and $j$ terms of collection.

Therefore, when it is desired to obtain a term $t$, belonging to Terms, new terms that are within the problem domain in question and to keep semantic proximity with $\mathrm{t}$, search for lines MSS is performed in order to identify line $t$ where it occurs. If this line exists, identifies the positions where are the biggest values it and the terms corresponding to them are selected as the greatest semantic similarity with the term. Can occur that $t$ is and then, establish adequate semantic relationships between search terms. This avoids misleading associations between terms - as the word "bank", for example, that in the context of financial investments keep a semantic proximity to the term "loan" but without any connection with the term "seat". The determination of the semantic proximity of terms is not a simple task. Factors such as synonymy (different words with the same meaning) and polysemy (same words with different meanings) difficult so much the automatic indexing terms semantically associated. The proposed model in this paper is illustrated, below in Figure 1. As it can be seen, when a term is submitted, the first step is to perform a query in the specific problem domain ontology under review. At this point, it looks up for classes (and associated subclasses) whose names feature in their formation the term to be enriched.As the chosen context to work with is the medical field, in particular cancers, ontology National Cancer Institute (NCI) was used.

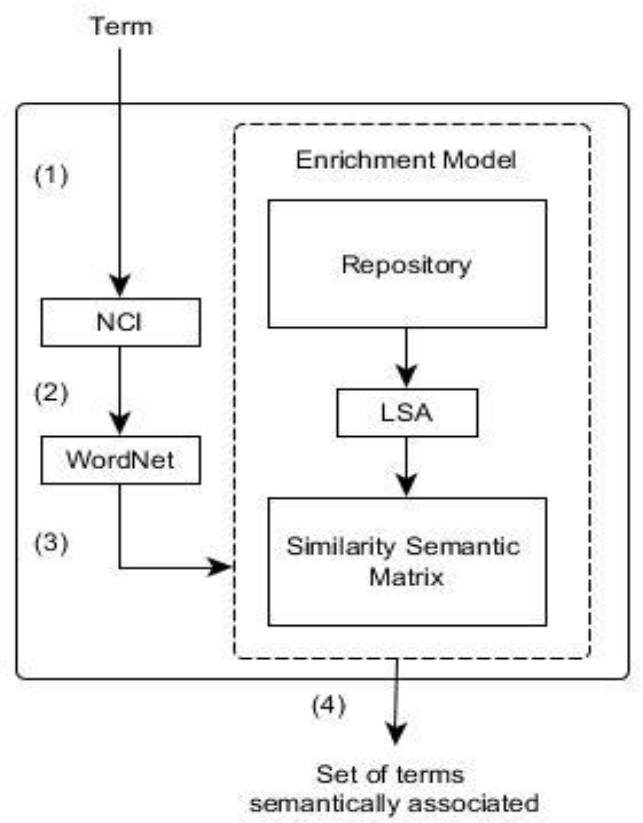

Fig. 2. Activity flow to the semantic enrichment

not found, but MSS have any synonyms of the term. For these cases, the WordNet is used to identify lines harboring synonymous terms, thus increasing the probability of finding semantically close to the original term.

As it could be seen, all the semantic enrichment process proposed here revolves around the semantic similarity matrix. Its construction is detailed in the next section.

\subsection{The Construction of semantic matrix}

\subsubsection{Construction of the repository}

The repository consists of a set of documents whose themes deal with particular subject. In this work, articles were selected from the medical field, especially dealing with issues related to brain cancer. So that the method developed here could be tested, it was decided to build the repository from freely accessible online articles form the magazine Plos One, published by the Public Library of Science. This magazine contains articles from the area of science disciplines and medicine and is indexed by entities such as PubMed and MEDLINE. As a large number of articles it is necessary, it was 
implemented a program to perform searches on the site, based on the International Classification of Diseases (ICD). This program also had the function to "clean up" the items taken from the site, eliminating, through regular expressions, html markup of the page views. As a result, the repository was built as a directory full of files in txt format containing only the words of each article.

\subsubsection{Latent semantic analysis}

To get the contextual relationship of words in text passages in repositories, it uses Latent Semantic Analysis (or), a statistical and mathematics technique able to eliminate the noise inherent in synonymy and polysemy [24]. The first step is to construct a matrix A, with $\mathrm{m}$ lines (number of words without repetition, constant in the repository) and $\mathrm{n}$ columns (amount of repository documents), called matrix-term document. Each Aij element of this matrix contains the numeric value relating to the importance of the term $\mathrm{i}$ in the $\mathrm{j}$ document, which is determined by a weight function. There are several implementation possibilities of weight function.

One of the most direct ways is the simple determination of the frequency of each term within a document. This approach has the disadvantage of assigning high values to words considered irrelevant, as articles pronouns and prepositions, very common in texts written in any language. Because of this, it is common to use the metric known as Term Frequency-Inverse Document Frequency (TF-IDF), which considers the frequency of a term $i$ in a certain document by dividing this amount by the number of documents within the repository $\mathrm{i}$ this term appears. Thus, if a tm term has high frequency in a certain document $d$, but also figure in almost all other documents repository, then tm present low weight value.But if this same tm does not appear so much in other documents, then its power to represent the document in the repository is high, which is reflected in its high value of TFIDF. Following construction of the matrix A, this is algebraically decomposed using the technique known as Decomposition in Singular Value - or Singular Value Decomposition (SVD) [25]. Thus, A becomes represented as the product of three other matrices:

\section{$\mathrm{A}=\mathrm{UDVT}$}

In Equation 1, $\mathrm{U}$ is a square matrix of dimensions $\mathrm{m}$ by $\mathrm{m}$, orthonormal, and its columns are singular vectors at left, $\mathrm{D}$ is an ordered diagonal matrix in decreasing order whose elements are called singular values and $\mathrm{V}$, as well as $\mathrm{U}$ are orthonormal, but with dimensions $\mathrm{n}$ by $\mathrm{n}$, with columns called as singular vectors at right. From A and to support the SVD method, it is possible to construct a second matrix Ak (Equation 2), which serves as approximation of the original matrix. So, it selects the larger $\mathrm{k}$ values of $\mathrm{D}$, yielding a matrix $\mathrm{Dk}$ dimensions $\mathrm{k}$ by $\mathrm{k}$, and their corresponding singular vectors $\mathrm{U}$ and $\mathrm{V}$, yielding a matrix $\mathrm{Uk}$, and $\mathrm{Vk}$ of dimensions $\mathrm{m}$ by $\mathrm{k}$ and $\mathrm{k}$ by $\mathrm{m}$, respectively. There is no accurate method to set the value of $k$, which leads to the empirical test for determination.

\section{$\mathrm{Ak}=\mathrm{UkDkVkT}$}

The practical effect of this process is to obtain a matrix with values free from noise, since that $\mathrm{A}$ is rebuilt based only in their most significant values. It is from $\mathrm{Ak}$ that all entire semantic analysis is done, since the problems caused by synonymy and the polysemic are much reduced when considering the approximate matrix. Furthermore, it should be noted that Ak has the same dimensions as A, thus, any term i of the original matrix is directly mapped in the reduced matrix, the same goes for each document $\mathrm{j}$.

\subsubsection{Construction of semantic similarity matrix.}

From the Ak matrix, it is possible to infer the degree of semantic proximity of any two terms in the repository. As each line of this matrix is a term and each column, a document, the lines Ak can be considered as vectors that locate each term in hyperspace of dimension $n$.

To determine the degree of proximity between two terms a and $\mathrm{b}$, we used a metric based on projections of vectors, as can be seen in Figure 3. Firstly, we identify the higher modulus vector - in this case, the vector $b$. Then it is projected the vector $b$ on the vector a, yielding the vector projection $\mathrm{p}$. The greater the projection module rises vector $b$, the greater the semantic proximity between them. As the magnitude of the projection of $\mathrm{a}$ on $\mathrm{b}$ is compared with the $\mathrm{b}$ module, the resulting numeric value is the percentage of $\mathrm{p}$ for $\mathrm{b}$. This process is applied to all pairs of terms Ak matrix, resulting in MSS.

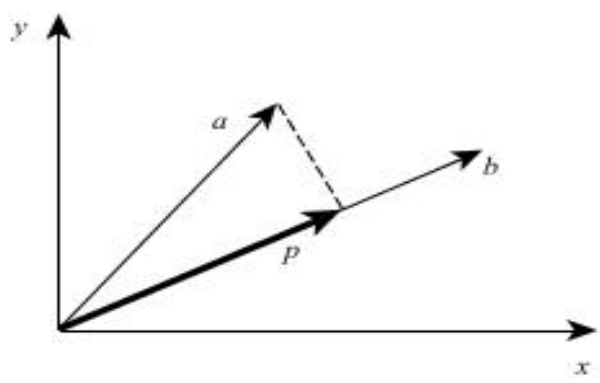

Fig. 3. Comparison between semantic terms based on vector projection

\section{RESULTS AND DISCUSSIONS}

The studies and surveys indicated in the previous sections were the basis for the development of an application that can implement the semantic enrichment according to the model. The program was developed in the Java programming language, as this natively supports the object-oriented programming and multiplatform execution. The repository used is composed of 1443 articles taken from the site Plos One, as discussed in section 5.2.1, which also discusses the criteria for selection of these documents.

The preparation stage of thermo-document matrix resulted in a matrix with 27644 lines (terms) by 1443 columns (documents). The SVD decomposition to obtain Ak was performed with $\mathrm{k}=$ 85.

As the data analysis requires considerable computational effort, especially when working with repositories with large number of documents, statistical information (such as absolute and relative frequencies of terms in documents, TF-IDF values of each term in each document, among others ) were persisted in a data base. In this case, we opted for the use of PostgreSQL, free and robust database, widely used today.

The experiments were performed on a computer equipped with 6 GB of RAM memory, Intel Core i-3 third-generation processor with clock frequency of $1.8 \mathrm{GHz}$ and operational system 7 Debian "Wheezy". The process of creating the semantic similarity matrix lasted three hours, approximately.

The tests were done at major fronts:

1. The comparison between queries enriched only by the use of the WordNet based synonyms and other similar words (heteronyms, hyponyms, etc.) and queries enriched by the 
proposed method in this paper. In both versions, was used to identify NCI ontology classes and subclasses corresponding appeared that the words of the search terms as input of the enrichment methods.

2. A comparison of results in search engines by the original query and the enriched query. In this case, to demonstrate the generality of the results, consultations were held a search engine that indexes lot of scholarly articles. The search term used for the tests was brain tumor. When subjected to enrichment WordNet means obtains the set of terms shown in Fig. 4.

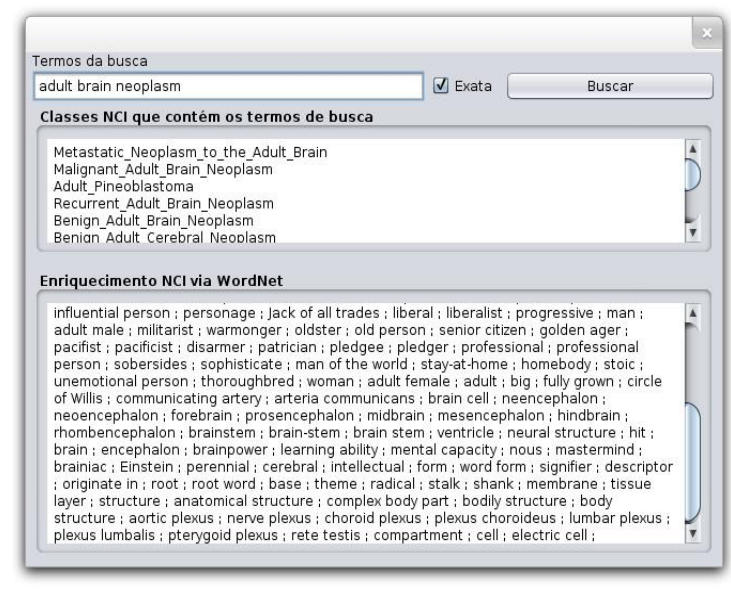

Fig. 4. Enrichment of brain tumor query by selecting similar words via WordNet. Note the presence of some relevant terms to the query context, like Einstein, for example.

It is noted the presence of large amounts of terms, some of them with low semantic relevance to the query context. For example, the word Einstein, reference to the great physicist, it is always associated to large brain capacities, but there is nothing to do with the context. In contrast, as can be seen in Fig. 5, when the same query is submitted to the proposed model in this article, based on the MSS, it obtains a significantly lower and more right contextual terms.

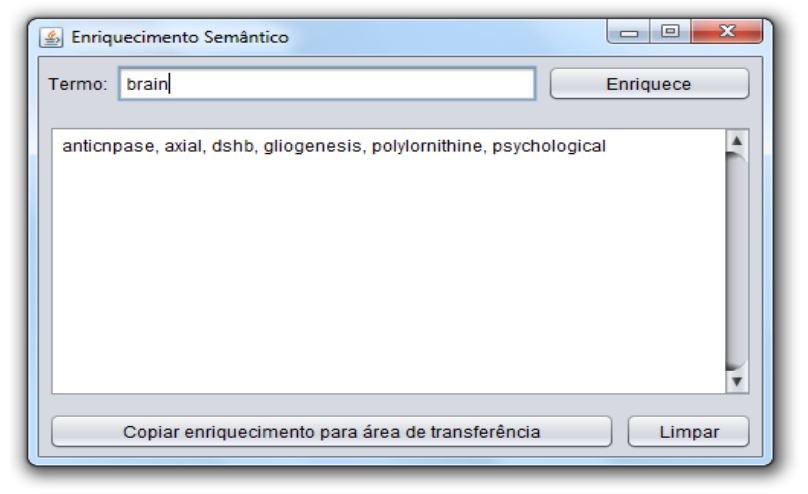

Fig 5. Enrichment via MSS. Note that, although there are a limited number of terms, these are better contextualized.

This phase of the tests is to compare the search for scientific articles by the enriched query. For this part, the PubMed was used, the platform that brings together specialized articles in the medical field. When the original term (brain tumor) appears to search, there is the recovery of 171286 articles, whose relevance should be analyzed by the professional who carried out the searching. When the same query is rather enriched by WordNet and subsequently submitted to the search platform, no result is achieved due to the large amount of terms and any degree of semantic variation of the terms of reference. Finally, when the query is submitted by MSS, PubMed shows a single result: the abstracts of the Seventh Congress of the European Association for Neuro-Oncology (EANO), held in 2006. When analyzing the document set therein, it is observed that the results are indeed contextualized with the desired search.

\subsection{General description of the method}

We chose to do the semantic enrichment of recovery because the searches do not get satisfactory results, with many documents in response or maintaining low quality of the so- called false positive results. The motivation for the development of this work is to address the specific problem of data recovery in the medical field, based on semantic enrichment method. Because the recovered information should be better addressed in that specific area because it will influence the strategies to be defined by doctors.

The choice to recover brain cancer information was due to research done in the SUS base of a Brazilian county, where it detected the huge number of people who had the brain cancer without a good return on its accomplishments treatments, which led us to think how we could improve the level of searches that would be performed by physicians because medical knowledge also have to be constantly recycled, to aid the diagnosis of disease.

With this information, began treatment for brain cancer research, which we used the NCI ontology, as one of the basis that assist in developing the project, and after this phase, is the following sequence performed for the semantic enrichment reaches its goals: 
1. Obtaining the texts to set the repositories

2. Statistical processing of the terms of each document

3. Implementation of the LSA

4. Generation of ontologies

5. Terms semantically associated.

\subsection{Precision and Recall}

In the initial experiment twenty queries were created from random fragments of medical documents obtained repository. Using each of the queries, comparisons were made using the proposed method and those indicated in the literature according to the text to be identified to obtain the best result.

Precision and recall have been extensively used to evaluate the retrieval performanceof IR algorithms. Considering

- I: an information request,

- $\quad \mathrm{R}$ : the set of relevant documents for I,

- A: the answer set for I, generated by an IR system

- $\quad \mathrm{R} \cap \mathrm{A}$ : the intersection of the sets $\mathrm{R}$ and $\mathrm{A}$.

Precision is the fraction of the relevant documents (the set R) which has been retrieved, i.e.,

$$
\text { Precision }=\frac{|R \cap A|}{|A|}
$$

Precision $=$ recovered relevant $/$ recovered

Recall is the fraction of the relevant documents (the set R) which has been retrieved,ie.,

$$
\text { Recall }=\frac{|R \cap A|}{|R|}
$$

\section{Recall= recovered relevant $/$ relevant}

In this experiment were considered answers valid documents with proximity greater than or equal to 0.20 (20 percent). And the obtained results were best in the proposed method.

Terms used for queries are presented in the Table 7.1 below:

Table7.1-Table consulted terms

\begin{tabular}{|c|c|}
\hline Consults & Terms \\
\hline 1 & Cancer \\
\hline 2 & Cancer of the brain \\
\hline 3 & Tumor Treatment \\
\hline
\end{tabular}

Experiments with results in the Table 7.2 below:

Table 7.2- Comparing the results of searches in precision

\begin{tabular}{|l|c|c|c|c|}
\hline Method & $\begin{array}{c}\text { Maia } \\
{[\mathbf{2 6}]}\end{array}$ & $\begin{array}{c}\text { Manning } \\
{[\mathbf{2 7}]}\end{array}$ & $\begin{array}{c}\text { Noveli } \\
{[\mathbf{2 8}]}\end{array}$ & $\begin{array}{c}\text { Araújo } \\
(\mathbf{2 0 1 5})\end{array}$ \\
\hline Test & Precision & Precision & Precision & Precision \\
\hline $001-005$ & 0,88 & 0,91 & 0,93 & 0,98 \\
\hline $006-010$ & 0,85 & 0,91 & 0,9 & 0,97 \\
\hline $011-015$ & 0,82 & 0,89 & 0,85 & 0,96 \\
\hline $015-020$ & 0,8 & 0,84 & 0,8 & 0,92 \\
\hline
\end{tabular}

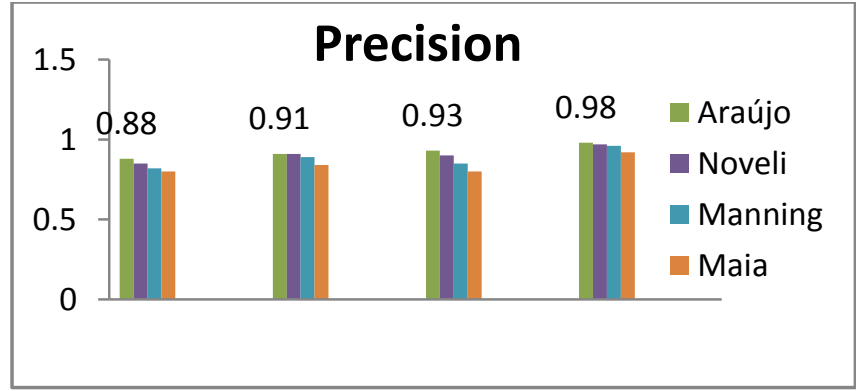

Fig.5 - Precision results in the graph

Experiments with results in the Table 7.3 below:

Table 7.3- Comparing the results of searches in recall

\begin{tabular}{|l|c|c|c|c|}
\hline Method & Maia & Manning & Noveli & Araújo \\
\hline Test & Recall & Recall & Recall & Recall \\
\hline $001-005$ & 0,87 & 0,89 & 0,91 & 0,97 \\
\hline $006-010$ & 0,85 & 0,88 & 0,87 & 0,95 \\
\hline $011-015$ & 0,79 & 0,79 & 0,82 & 0,91 \\
\hline $015-020$ & 0,77 & 0,76 & 0,8 & 0,9 \\
\hline
\end{tabular}

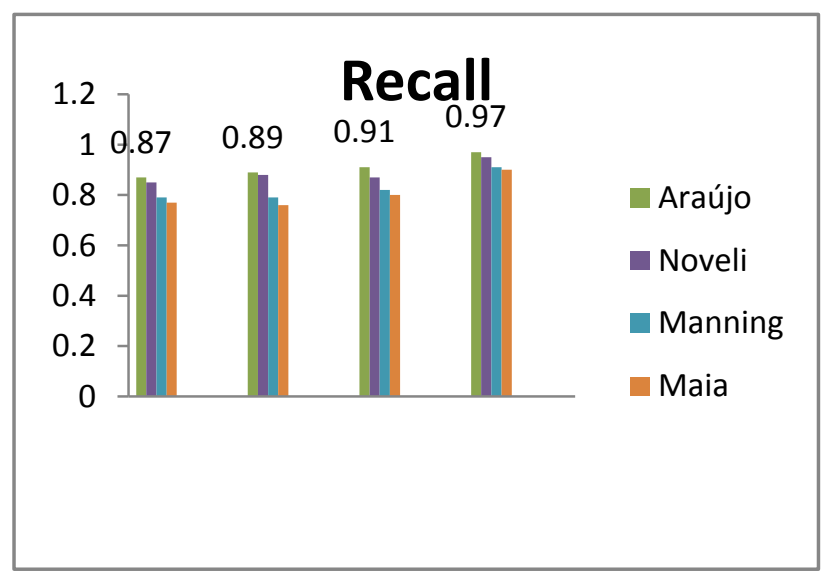

Fig.6 - Recall results in the graph

The test results responded to expectations, providing precise relationship about the types of items retrieved during the search, in relation to other algorithms tested.

\section{CONCLUSION}

In this paper, we presented a simple model of semantic enrichment that was able to produce results within specific contexts. This leads to better search results and brings to important medical advances of the web-semantic field.

The need to produce results that take into account not only the syntax but also the semantics of query terms is currently the subject of great importance in the area of scientific research in various fields of knowledge. The application of this technique in the medical field can bring great benefits to professionals and patients. So, in summary the research problem of this proposal is linked to the search for a consistent way to retrieve semantically relevant medical articles based on clinical data, and how this data can be recovered in dynamically and automatically compared the searches performed by users that area.

However, we can use the semantic enrichment method proposed to be used in searches for articles in other medical 
areas, such as for patients with chronic diseases, can also be enriched information for other types of malignant neoplasms (cancer) so it would also be necessary to use a specific ontology for each aforementioned situation, which will cause new areas of research, which can achieve a recovery more consistent information regarding informal learning in the medical field.

\section{ACKNOWLEDGMENT}

The authors thank CAPES, the ITA and the IFG, the partnership that made possible the realization of the project DINTER

\section{REFERENCES}

[1] Yan, P., Jiao, Y., Hurson, A. R. e Potok, T. E. 2006. Semantic-based information retrieval of biomedical data In Proceedings of the 2006 ACM Symposium on Applied Computing, New York, USA, pp 1700-1704.

[2] Liu, J. and Birnbaum, L. 2007. Measuring Semantic Similarity between Named Entities by Searching the Web Directory. In Proceedings of the IEEE/WIC/ACM international Conference on Web intelligence. Washington-DC, USA, pp 461-465.

[3] Soergel, D. 2005. Thesauri and ontologies in digital libraries. In Proceedings of the 5th ACM/IEEE-CS Joint Conference on Digital Libraries. New York, USA, p 421.

[4] Keleberda, I., Repka, V., e Biletskiy, Y. 2006. Building learner's ontologies to assist personalized search of learning objects. In Proceedings of the 8th international Conference on Electronic Commerce. Fredericton, New Brunswick, Canada, pp 569-573.

[5] Berners-Lee, T., Hendler, J. Lassila, O. The Semantic Web. Scientific American., n. 5, p. 29-37, 2001.

[6] Gruber, T., 1993. A translation approach to portable ontology specification. Knowledge Acquisition Journal, Vol 5, No. 2 pp 199-220.

[7] Finin, T., Ding L., Zou L. 2005.Social Networking on the Semantic Web-University of Maryland, Baltimore County Baltimore MD USA.

[8] Breitman, K.K. 2005. Web Semântica: a Internet do futuro. Editota LTC. Rio de Janeiro, Brasil.

[9] Miller, G., Beckwith, R., Fellbaum, C., Gross, D., Miller, K., 1990. Introduction to WordNet: An On-line lexical Database. International Journal of Lexicography, 3(4), pp. 235-244.

[10] Richardson, R., Smeaton, A. F., 1995. Using WordNet in a Knowledge-Based Approach to Information Retrieval. University of Dublin technical report CA-0395, Dublin, Ireland

[11] Smeaton, A. F., Quigley, I., 1996. Experiments on Using Semantic Distances Between Words in Image Caption Retrieval. University of Dublin technical report CA0196, Dublin, Ireland.

[12] Gómez-Hidalgo, J.M. and Rodriguez, M.B. 1997. Integrating a Lexical Database and a Training Collection for Text Categorization. ACL/EACL Workshop on Automatic Extraction of Lexical Semantic Resources for NL Applications. Madrid, Spain, pp. 39-44.

[13] Miller, G. A., 1985. 'Wordnet: A Dictionary Browser' in Information in Data, Proceedings of the First Conference of the UW Centre for the New Oxford Dictionary. University of Waterloo. Waterloo, Canada.

[14] Grossman, D. A.; Frieder, O. 2004. Information Retrieval: Algorithms and Heuristics. [S.1.]: Dordrecht, Netherlands : Springer, 272p.

[15] Salton,G.M., McGill, M.J. 1983. "Introduction to Modern Information Retrieval." McGraw-Hill, Michigan,USA, 1983 ISBN 0070544840, 1983

[16] Shortliffe, E. H., Barnet, G. O. 2001. Medical data: Their acquisition, storage and use. In: Shortliffe, E. H.; Perreault, L. E.; Wiederhold, G.; Fagan, L. M (Ed.). Medical informatics computer applications in health care and biomedicine. 2nd ed. New York: Springer.

[17] Lin, K. H.-Y.; Hou, W.-J.; Chen, H.-H. 2005. Retrieval of Biomedical Documents by Prioritizing Key Phrases In: Proceedings of the 14th Text REtrieval Conference, Gaithersburg, Maryland.

[18] Mykowiecka, A.; Marciniak, M.; Kupsc, A. 2009. Rulebased information extraction from patients' clinical data J. of Biomedical Informatics, v. 42, n. 5, p. 923-936.

[19] Lourenço, A.; Carreira, R.; Glez-Peña, D; Méndez, J. R.; Carneiro, S.; Rocha, L. M.; Díaz, F.; Ferreira, E. C.; Rocha, I.; Fdez-Riverola, F.; Rocha, M. 2010. BioDR: Semanticindexing networks for biomedical document retrieval. Expert Systems with Applications, v. 37, n. 4, p. $3444-3453$

[20] Thakker, D. et al., 2012.Taming Digital Traces for Informal Learning: A Semantic-driven Approach., Berlin, Heidelberg. Anais. Berlin, Heidelberg: SpringerVerlag, pp.348-362.

[21] Baldan, M. A., Menezes, C. S. 2012. Um Ambiente para Construção de Perfis a Partir de Textos Pessoais. Anais do Simpósio Brasileiro de Informática na Educação, v. 23 , n. 1

[22] Zapater, J. J. S., Mendes Neto, F. M., 2014. Uso de tecnologías semánticas en diferentes dominios de aplicación: Entorno educativo y sistemas de información de tráfico vial. Saarbrücken: Editorial Académica Española, Madrid,Espanha.

[23] Sheth, A., Arpinar, I. B., Kashyap, V., 2003 Relationships at the heart of semantic web: Modeling, discovering, and exploiting complex semantic relationships. SpringerVerlag, p p. 63-94.

[24] Landauner ,T., Foltz W. P., Laham D.,1998. "Introduction to Latent Semantic Analysis", Lawrence Erlbaum Associates, New Jersey, USA.

[25] Golub, Gene H.; Van Loan, Charles F., 1996. In: Gene H.. Matrix Computations. Vol 3, JHU Press, Baltimore, USA.

[26] Novelli, A. D. P.; Oliveira, J. Simple method for ontology automatic extraction from documents. International Journal of Advanced Computer Science and Applications, v. 3, p. 44-51, 2012.

[27] Manning, C.; Raghavan P.; Schutze, H.An Introduction to Information Retrieval. New York: Cambridge University Press, 2009

[28] Maia, L. G.; Souza, R. R. Medidas de similaridade entre documentos eletrônicos. IX ENACIB, 2008. 